\title{
The Origin of Nonlinear Dynamics Involving Complexity in Modern Sciences
}

\author{
Deok-Soo Cha ${ }^{1 *}$, Hee Jong Jun ${ }^{2}$ \\ ${ }^{1}$ Eho Technology Co., Soongsil University, Seoul, Korea \\ ${ }^{2}$ Soongsil University, Seoul, Korea \\ Email: *chdsoo@hotmail.com
}

How to cite this paper: Cha, D.-S. and Jun, H.J. (2020) The Origin of Nonlinear Dynamics Involving Complexity in Modern Sciences. Open Journal of Applied Sciences, 10, 654-662.

https://doi.org/10.4236/ojapps.2020.1010045

Received: August 24, 2020

Accepted: October 26, 2020

Published: October 29, 2020

Copyright $\odot 2020$ by author(s) and Scientific Research Publishing Inc. This work is licensed under the Creative Commons Attribution International License (CC BY 4.0).

http://creativecommons.org/licenses/by/4.0/ (c) (i) Open Access

\begin{abstract}
This paper reveals the origin of nonlinear dynamics and presents a solution for nonlinear systematic problems based on other science. Generally, physical phenomena are divided into linear static logical problems and nonlinear dynamic systematic problems, but all scientists have solved both problems using the same algebraic logical solution in statistical physics based on determinism such as chaos theory. Surprisingly, this is a contradiction and a serious mistake because there is a perfect solution such as the system analysis theory existing in other science. Unfortunately, it has developed in the $20^{\text {th }}$ century by engineers. Thus, classical physicists could not solve it. Meanwhile, the author achieved the systematic solution for many unsolved nonlinear systematical, further, proved the research result through simulation using specially designed simulation device. Thus, this is a revolutionary achievement because it can easily solve the unsolved nonlinear dynamics that exists in all fields of science. Ironically most determinists do not welcome and reject it. However, it has no matter, it will be separated from current physics and other scientists studied it in the second physics. Therefore, it would be contributed to solve the unsolved nonlinear dynamics in complex science.
\end{abstract}

\section{Keywords}

Nonlinear Dynamics, Indeterminism, MATLAB, Control Theory

\section{Introduction}

This paper addresses nonlinear dynamic systematical problems involving complexity [1] that have not been solved until now, excluding materials science. While these interdisciplinary studies have been solved by physicists, using statistical physics based on determinism such as chaos theory, these problems have 
not yet been clarified. This is because most physicists misconstrue that irregularities in these problems are permanent inherent properties and impossible to solve. In practice, there is no perfect solution. However, this study will provide a new perfect solution based on technological science and reveal the origin of nonlinear dynamics in physical science.

While conducting another research in 2015, the author surprisingly discovered a serious hidden problem in classical physics that arose three hundred years ago. However, physicists cannot explain the reason behind it, and they avoid the question. Thus, it is essential to consider the reason here. Generally, the paradigms of science can be divided into determinism and non-determinism. Likewise, physical phenomena are divided into two groups; linear static logical problems and nonlinear dynamic systematic problems, as shown in Figure 1(a) and Figure $1(b)$. However, there is no perfect systematic solution in modern science, so they have no choice. Therefore, all scientists have solved both the problems using the same algebraic logical solution in statistical physics based on determinism, such as chaos theory.

Surprisingly, it is a grave mistake as a contradiction in science. Furthermore, it is shocking issue because there is already existed a perfect solution in existing technological science, such as the systems analysis theory in control theory [2], as shown in Figure 3(a). If this is true, physicists need not work hard anymore to solve nonlinear systematic problems in the future; moreover, it could be a revolutionary achievement similar to the Copernican theory. If this is indeed true, we wonder why classical physicists in the $17^{\text {th }}$ century could not clarify the contradiction as mentioned above. Ironically, it is contrary to determinism. Thus, determinists still reject the above solution because they have no regard with other sciences

Nevertheless, in 2015, the author has completed the solution based on the control theory and published it [3] [4]. However, they do not dislike to expose the above contradiction, further, they hope keeping existing physics based on determinism. Hence, the author failed to obtain their consent. Thus, the author disappointed because they did not want innovation. However, there is no problem.
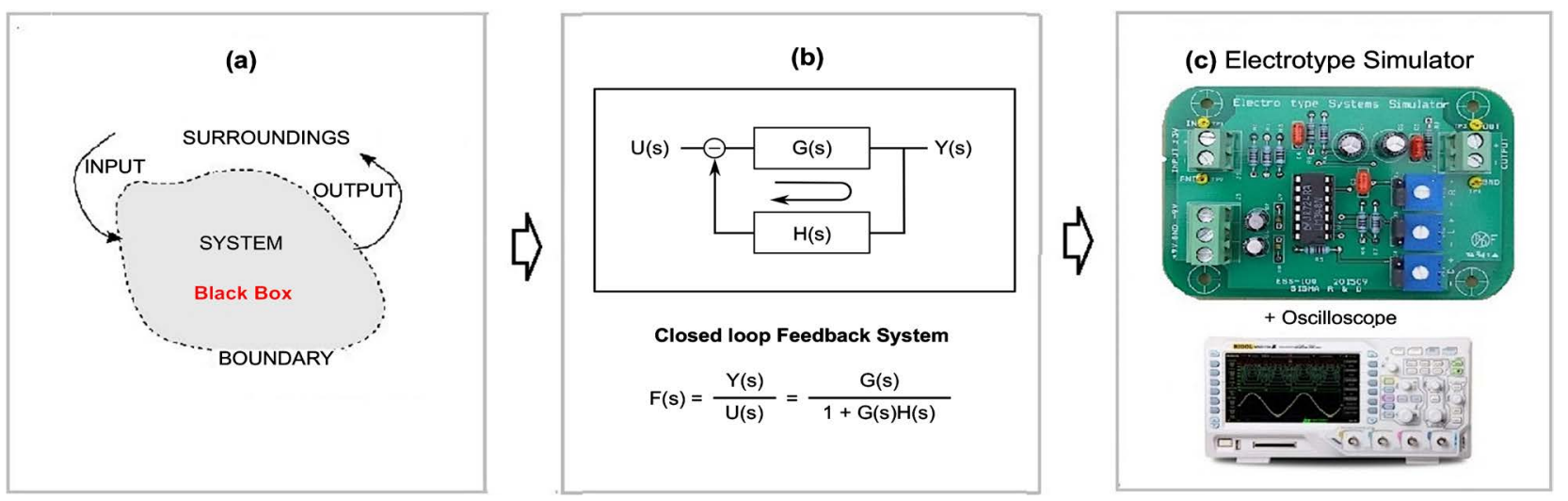

Figure 1. (a) Modern science treated the physical problems as an invisible black box and analyzed using logical solution based on determinism. (b) The internal mechanism of nonlinear systematic problems, the basic systems can be defined as a closed loop system with feedback element. (c) Electrotype analog simulator (equivalent feedback system). 
Astonishingly, in 2019, the author obtained another research result; 1) determinists have no relation to the above solution because it is completed based in indeterminism, not cur-rent physics. 2) The solution needs not approval and verification from determinists. Because, the result was solved and proven through simulation with a specially designed simulator (see Figure 1(c) and Appendix), anyone can reproduce the result and confirm it through the simulation device as mentioned above. The other word, the device is similar to Kepler's helioscope, which proved that the Earth is round, or Wright's airplane, which proved that humans could fly. Hence, if someone wants to confirm the result, they are encouraged to simulate it.

Nevertheless, most physicists misunderstand that the result is not advantage to them similar to the Copernican theory, hence, they do not welcome it. Ironically, Moreover, most physicists misunderstand the new solution is a part of deterministic physics, moreover, they attempt to solve with deterministic physics. Therefore, the new solution is established as the second physics to distinguish it from the existing first physics. If so, they need to clarify the following issues about control theory;

- What is systems analysis theory in control theory?

- Why are classical physicists unable to solve these problems?

- What is the origin of nonlinear dynamics in complexity?

For those who doubt the validity of this solution, there are two cases of practical application. Although they are different from what the scientific community may have known in the past, they are certain. The author hopes to clarify the understanding in this regard.

\section{Methods and Materials}

\subsection{Scientific Background}

First, we need to look back to classical physics based on determinism. Why can they not find the above perfect solution, such as the systems analysis theory? Unfortunately, they misunderstood that systematic nonlinear problems are treated as algebraic logical problems, they did not know other solution. Ironically, the systems analysis theory in control theory [2] has developed in the $20^{\text {th }}$ century for designing automatic systems by engineers such as Nyquist; however, classical physicists cannot know the principle. Paradoxically, if the above control theory was appeared earlier, classical physicists, including Newton, could have cleared the above contradiction. Thus, they have no choice because there is no other solution. Nevertheless, all determinists strongly reject the control theory and prefer their old deterministic physics. However, other scientists have nothing to do with physicists. If physicists do not clarify and leave the contradiction in modern science, non-physicists have to solve the nonlinear systematic problems instead of them. Therefore, physicists have no choice. In other words, it will be separated from current physics and transfer to another science, such as the second physics, which will emerge as an independent science based on the 
paradigm of indeterminism. On the other hand, if physicists would accept this solution, they can utilize both old and new solutions and they can solve many unsolved nonlinear dynamics, including metaphysical problems in holistic science fields.

\subsection{The Origin of Nonlinear Dynamics}

There is something to watch out for. Many physicists misconceive the solution as deterministic physics, it must be solved by control theory involving indeterminism. They need to study the mathematical tool in control theory based on the Laplace transform, which is as difficult as chaos theory for all scientists. Hence, it must be supported by a MATLAB program [5] or analog type simulator (refer to Appendix). To help readers understand, the author would like to explain it with a drawing.

Normally, input source is a random function in Figure 2(a) but the inherent system properties is determined as shown in Figure 2(b), the output overlaps with both properties, as shown in Figure 2(c); it has organized irregularities. In addition, this study is simulated by the above device instead of a mathematical process. Further, anyone must follow four steps: system modeling-simulation - verification-return. However, not even knowing the control theory, they are able to solve and confirm it using a computer program.

- (First step: Modeling) In an example of a food chain in ecology, physicists have treated the problems as a black box, as shown in Figure 1(a). First, we need to build a model system, which is a closed-loop system with a feedback element, as shown in Figure 1(b). Thus, the output of the model systems will converge to an equilibrium state. Figure 1 (c) is a novel simulation device as an invention, which can simulate any type of feedback system by anyone. Surprisingly, it has an equivalent system in Figure 1(b) with the same parameter as Equation (1) [4].

$$
F(s)=\frac{Y(s)}{U(s)}=\frac{G(s)}{1+G(s) H(s)}=\frac{\omega^{2}}{s^{2}+2 \beta \omega s+\omega^{2}}
$$

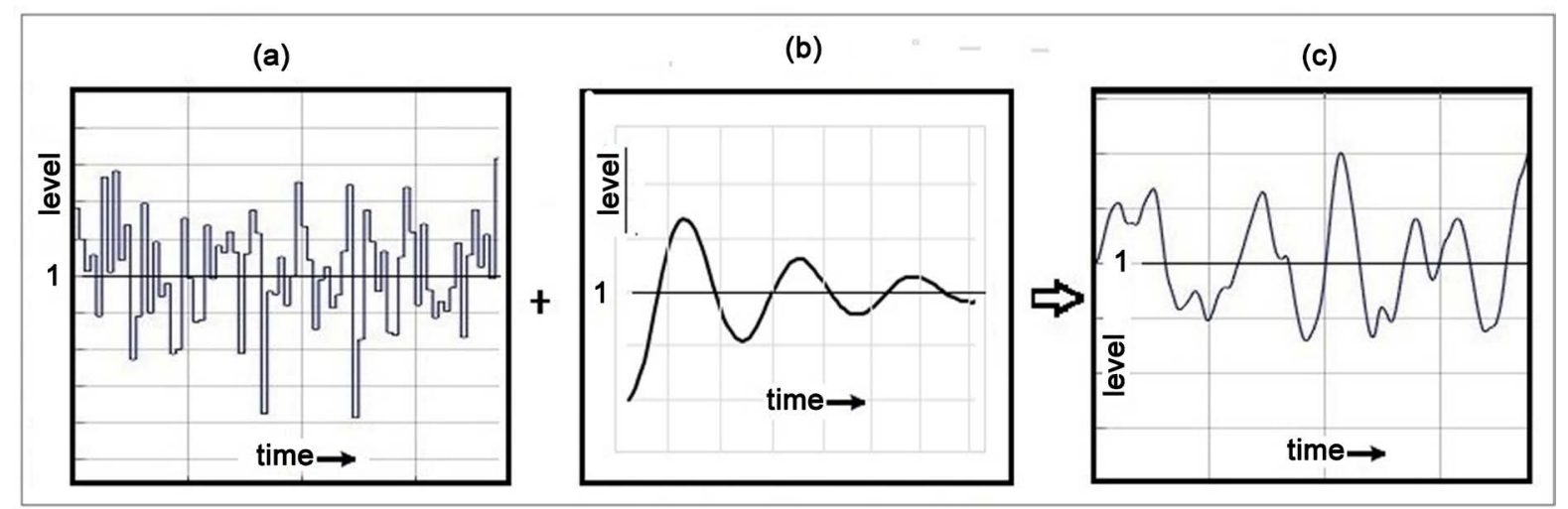

Figure 2. (a) Behavior of input is randomizing data; (b) Inherent characteristics of systems as transfer function $\mathrm{F}(\mathrm{s})$; (c) the output is overlapped with input and systems function $\mathrm{F}(\mathrm{s})$. 
(a) Control Theory Textbook

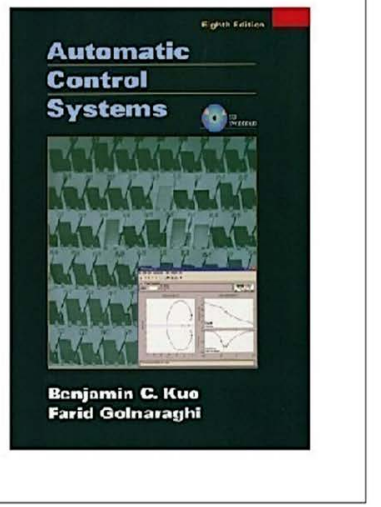

where $[s]$ is the Laplace operator, $U(s)$ is the input source, $Y(s)$ is the output function, $\omega$ is a constant, and $\beta$ is the damping factor. The damping factor determines the peculiar characteristics of all feedback systems; it depends on the damping factor; the intrinsic characteristics vary as shown in Figure 3(b). The practical output $y(t)$ of the basic input is determined by Equation (2), which has a decreasing periodicity and converges into a stable saturation.

- (Second step: Simulation) For readers' understanding, assume that a virtual system exists and Equation (1) is determined as follows: it is the most common case. If you want, you can use arbitrary functions.

$$
y(t)=1-A \cdot \mathrm{e}^{-B \cdot t} \sin (W \cdot t+\varphi)
$$

where $\omega=1$ and $\beta=0.2$, we put this data into a computer and begin to simulate. It can reproduce the behaviors of nonlinear dynamics phenomena on the computer screen, as shown in Figure 3(c). It is a response of the random source in real time from MATLAB. Likewise, anyone can observe the results in real time, as shown in Figure 4(c).

$$
F(t)=1 /\left(s^{2}+0.4 s+1\right)
$$

- (Third step: Verification) Anyone can experiment with any type of systematic problem because the stimulator has several adjustable controllers. Therefore, the physical black box in the nonlinear systematic problem is solvable. Please watch the video clip [6].

\subsection{Practical Analysis of Nonlinear Dynamics}

- (Hypothesis) Most physicists have been treated the food chain in ecology as a black box. If the black box can be defined as a closed loop system, as shown in Figure 1(b). In this case, the number of individuals of predators is forced to be limited by the number of individuals of prey; in the case of the stock market, buyers, and sellers. If so, it can analyze the correlation based on the systems analysis theory in real time.

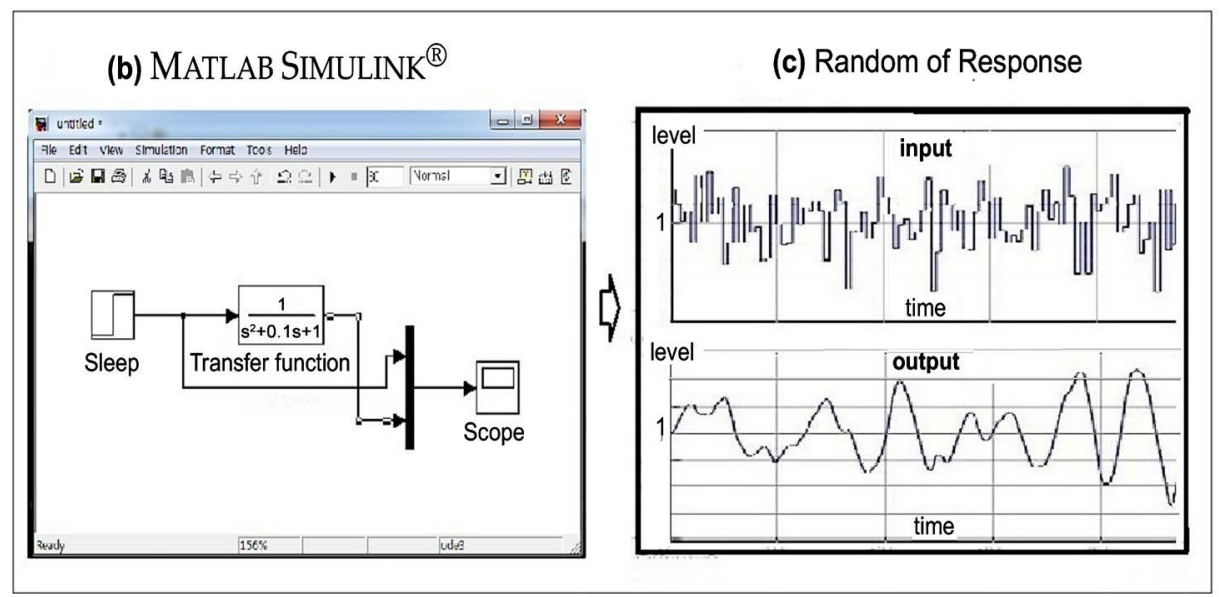

Figure 3. (a) Control theory textbook including systems analysis theory; (b) Commercial program MATLAB; (c) Simulation result-Response of random function by MATLAB. 

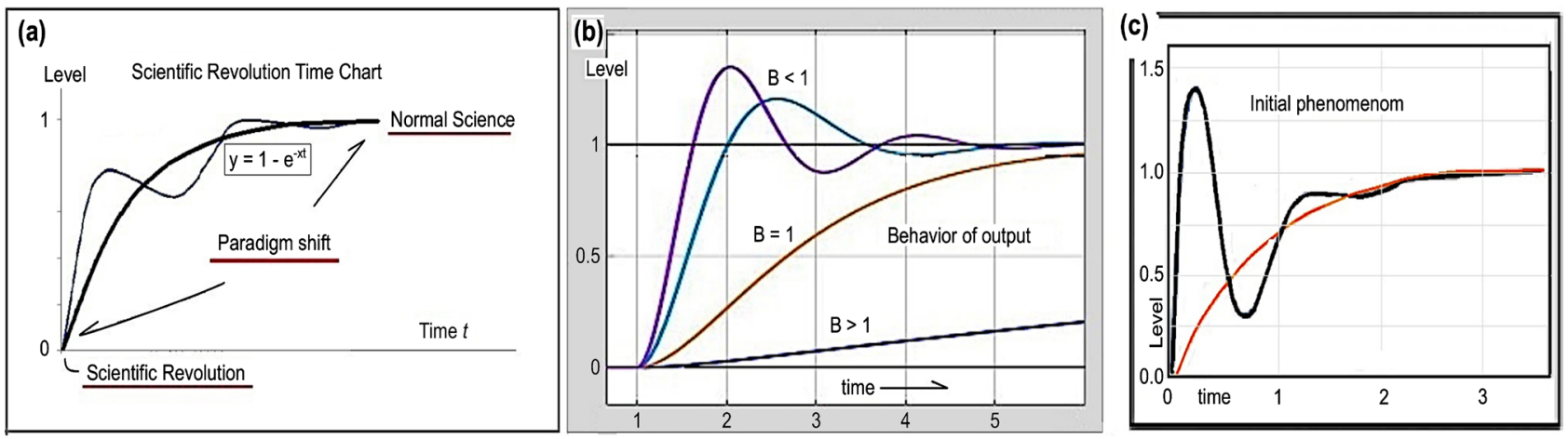

Figure 4. (a) Time chart of Kuhn's Scientific Revolution: Scientific Revolution-Paradigm Shift-Normal Science; (b) Basic Response of Output according to Damping Factor; (c) Response of Random Function in Simulator Display.

- (Simulation), we can simulate the above condition using a simulation device, as shown in Figure 1(c). If a random source is added to the computer program MATLAB with temporary parameter Equation (3), the output behavior, such as the amount of predator and prey or the daily stock price, will be displayed as shown in Figure 2(c). Therefore, it has nonlinear dynamics. We determined the origin of nonlinear dynamics in the process; it is derived from closed loop systematic problems.

- (Verification) If true, nonlinear dynamics do not occur by chance. Surprisingly, we found the behaviors of complexity in Figure 2(c), which overlapped with irregularities, fractal, self-organization, and initial phenomenon; moreover, it is never overflow or runaway, explosive. In addition, if readers can fully understand the new solution as mentioned above, they can understand the two practical applications as blow

\subsection{Practical Applications; Redefinition of Lorenz's Butterfly-Effect}

The butterfly effect was first claimed in 1963 by Edward Norton Lorenz [7], who was not a physicist but a meteorologist. Finding that a small numerical variation $(0.506127>0.5016)$ in an iterative calculation process in a weather forecast led to a completely different result, he named it as the butterfly effect, which made an appeal to the public and was welcomed by many determinists.

Unfortunately, the meteorologist Lorenz misunderstood the initial phenomenon in his own experiment, as shown in Figure 3(c), which was similar to an electrical impulse. It appears in science when the damping factor in Equation (1) is less than zero. Thus, it is rapidly increasing and overshooting, as shown in Figure 2(b). Therefore, it is too a time-series function, which can be confirmed by MATLAB. Paradoxically, if the meteorologist Lorenz knew the reason, he would not have made the assertion. Surprisingly, his assertion is an unreasonable inference like a fiction, an overstated idea, or an illusion. At any time, anyone can reproduce the phenomenon using a simulation device. No technological scientists believe mysticism in modern science. 


\subsection{Practical Applications; Redefinition of Kuhn's Innovation Theory}

Kuhn conceptualized the history of technical invention [8]; he asserted that a scientific innovation occurs in the following three steps: scientific revolution-paradigm shift-normal science. The author describes the time chart in Figure 4(a).

(Modeling) For instance, gunpowder was invented in 1880. At that time, it was like a revolution. Its utility increased as soon as the war broke out. Therefore, people spent a huge amount of money for restoration. Repeatedly, the demand increased as another war broke out; he defined it as a paradigm shift. Finally, it was saturated and settled into a normal state. Ironically, it can be defined as a systematic problem, and the output can be determined as a time series function in Equation (2) above.

(Simulation and verification) We can obtain an exponential curve as shown in Figure 4(a) from the simulation device as shown in Figure 3(b) or Figure 3(c). There are also many examples such as automobiles, televisions, fertilizers, and computers. Unfortunately, he misunderstood a time series function as a philosophical theory. Ironically, Kuhn's theory is only a systematic problem. If he knew the reason, he would have not asserted it.

\section{Result}

This reveals that the origin of nonlinear systematic problems involving complexity is originated from closed-loop systems with feedback, as shown in Figure 1 (b).

- These nonlinear systems cannot be solved perfectly by logical algebraic solution in statistical physics. Instead, it must be solved by the systems analysis theory.

- If physicists reject the solution continuously, it must be separated from modern science and studied through second physics.

\section{Discussions}

The main objective of this paper is to reveal the origin of nonlinear dynamics, further, this study provides to physicists and non-physicists a perfect solution as control theory including a novel simulation device based on technological theory as the second physics. In addition, the author informs to regret to physicists that there is a contradiction in current physics. Nevertheless, non-physicists will clear the contradiction unless physicists do not clarify the problems because other scientists have no reason to follow their deterministic physics. Surprisingly, these problems can be easily solved by the commercial program MATLAB and analog simulation device.

Thus, no physicists need not hard work for many unsolved nonlinear systematic problems in science, such as food chain or stock price involving complexity. Perhaps, in the future, it will establish the second physics based on indeter- 
minism. It is a reasonable idea without risk because we can throw out the solution if it is not perfect; all scientists can study the discipline alone without physicist's interference. In addition, other scientists have no reason to reject the discipline because in the future, the solution would be solved the nonlinear systematic problems involving complexity, such as daily weather, daily stock price, ice age, quantum mechanics, turbulence, nuclear reactor dynamics without physicist's support, further, they need not hard work for the nonlinear systematic problem in their discipline in the future.

Therefore, physicists should be adopted this solution in the future. In particular, the author believes that this solution is a very valuable result for scientific institutes and organizations. An example, in the Santa Fe Institute [8] in USA, the results could be used to study for complexity in their mission. Perhaps, they need not hard work for complexity no more. This study could be essential and of significant assistance to their research.

\section{Conclusions}

This study presents the origin of nonlinear dynamic systems which are generated from closed-loop feedback systems; it is an inherent property. It should be solved by systematic solution, otherwise, it is out of basic principle as a contradiction. Especially, non-physicists are encouraged to review this research result; it will be easy to solve their unsolved nonlinear dynamic systematic problems, including metaphysical problems in science without chaos theory. However, the new perfect solution is different from existing algebraic logical solutions in the present first physics. It will be classified in the second physics in the future.

On the other hand, this study provides two examples of applications. Thus, it can be an innovative breakthrough in science, which is solved based on nonlinear dynamics. Notably, the solution is more elegant, precise, and easier compared with the old chaos theory. Nonlinear dynamic systems must be solved using the above systems analysis theory. Finally, this new solution contributes to the advancement and development of modern science.

\section{Acknowledgments}

Eho Technology Co. research center supported research.

\section{Conflicts of Interest}

The authors declare no conflicts of interest regarding the publication of this paper.

\section{References}

[1] Casti, J.L. (1995) Complexification: Explaining a Paradoxical World through the Science of Surprise. Harper Perennial, New York.

[2] Golnaraghi, F. and Kuo, B.C. (1998) Automatic Control Systems. 10th Edition, McGraw-Hill Education, New York.

[3] Cha, D.-S. (2015) Establishment of New Solution for Complex Systems in Multidis- 
ciplinary Science Based on Feedback System Analysis Method and Proven by Simulator. Journal of Modern Physics, 6, 1927-1934.

https://doi.org/10.4236/jmp.2015.613198

https://www.scirp.org/journal/paperinformation.aspx?paperid $=60738$

[4] Cha, D.-S. (2016) Complex Systems Are Not Black Boxes but Solvable Systematical Problems; Proven by Simulation and New Conception. Journal of Modern Physics, 7, 1540-1545. https://doi.org/10.4236/jmp.2016.712140 https://www.scirp.org/journal/paperinformation.aspx?paperid=70204

[5] MATLAB. https://kr.mathworks.com/support/learn-with-matlab-tutorials.html?s tid=srchtitle

[6] Video clip in YouTube. https://www.youtube.com/watch?v=-EnU4L5uH5o

[7] Lorenz, E. (1963) Deterministic Non periodic Flow. Journal of the Atmospheric Sciences, 20, 134-141.

https://journals.ametsoc.org/doi/abs/10.1175/1520-0469\%281963\%29020\%3C0130 \%3ADNF\%3E2.0.CO\%3B2 https://doi.org/10.1175/1520-0469(1963)020<0130:DNF>2.0.CO;2

[8] Santa Fe Institute. https://santafe.edu/

\section{Appendix; Design of Systems Simulator}

The author releases the creation such as simulation device to other scientists [3], which ingeniously consists of three OPAMP, a differentiator, an integrator, and a comparator for the first time. Then, its transfer function is determined as follow.

If the integrator is defined as $G(s)=\frac{1}{L C s^{2}}$ and the differentiator is $H(s)=1+R C s$. Substituting this function into basic equation $\frac{G(s)}{1-G(s) H(s)}$, we can obtain Equation (3). Finally, it is equivalent to Equation (1). Then, we succeeded in making simulator through Equation..

$$
F(s)=\frac{G(s)}{1+G(s) H(s)}=\frac{\frac{1}{L C s^{2}}}{1+\frac{1}{L C s^{2}}(1+R C s)}=\frac{\frac{1}{L C}}{s^{2}+\frac{R}{L} s+\frac{1}{L C}}
$$

If someone adjusted the variable $R, L, C$ in Equation (3), they can produce any type feedback systems such as Figure 2(c); then, it is very perfect and precise and easier than chaos theory in statistical physics 